\title{
Evaluation of the Segmentation by Multispectral Fusion Approach with Adaptive Operators: Application to Medical Images
}

\author{
Lamiche Chaabane \\ Department of Computer Science \\ University of M'sila \\ Algeria
}

\begin{abstract}
With the development of acquisition image techniques, more and more image data from different sources of image become available. Multi-modality image fusion seeks to combine information from different images to obtain more inferences than can be derived from a single modality. In medical imaging based application fields, image fusion has emerged as a promising research area since the end of the last century. The paper presents the evaluation of the segmentation of MR images using the multispectral fusion approach in the possibility theory context . Some results are presented and discussed.
\end{abstract}

Keywords- image fusion; possibility theory; segmentation; MR images.

\section{INTRODUCTION}

Magnetic resonance (MR) imaging has been widely applied in biological research and diagnostics, primarily because of its excellent soft tissue contrast, non-invasive character, high spatial resolution and easy slice selection at any orientation. In many applications, its segmentation plays an important role on the following sides: (a) identifying anatomical areas of interest for diagnosis, treatment, or surgery planning paradigms; (b) preprocessing for multimodality image registration; and (c) improved correlation of anatomical areas of interest with localized functional metrics [1].

Segmenting MR images has been found a quite hard problem due to the existence of image noise, partial volume effects, the presence of smoothly varying intensity inhomogeneity, and large amounts of data to be processed. To handle these difficulties, a large number of approaches have been studied, including fuzzy logic methods [2], neural networks [3], Markov random field methods with the maximum expectation [4], statistical methods [4], and data fusion methods [5], to name a few.

As one typical data fusion problem, the segmentation of multi-modality brain MR images aims at achieving improved segmentation performance by taking advantage of redundancy and complementariness in information provided by multiple sources. There have existed many data fusion methodologies, which are capable of reasoning under various types of uncertainty. Typical ones include probability theory based approaches, possibility theory based approaches, and Dempster-Shafer evidence theory based approaches [6].

\author{
Moussaoui Abdelouahab \\ Department of Computer Science \\ University of Setif \\ Algeria
}

Traditionally probabilities theory was the primary model used to deal with uncertainty problems, but they suffer from drawbacks which are still a matter of discussion. Whereas the Dempster-Shafer theory also allows to representing these two natures of information using functions of mass but the set of operators used by this theory in fusion step is very restricted.

Alternative to this approach is the possibility theory where uncertainty and imprecision are easily modeled, in this article we will focus on this last one for two essential reasons : this theory allows to combining information coming from various sources by the use a wide range of available combination operators. In addition, this theory seems to us the most adapted to the considered problem in the modeling step [1][6].

In this work we aim to evaluate the segmentation of the human brain tissues using a multispectral fusion approach. This approach consists of the computation of fuzzy tissue maps in each of two modalities of MR images namely T2 and PD as an information source, the creation of fuzzy maps by a combination operator and a segmented image is computed in decision step.

The remainder of the paper is organized as follows : In section II, some previous related works are briefly cited. section III describes the FPCM algorithm. In section IV, we present the principals of possibility theory reasoning . Section $\mathrm{V}$ outlined the fusion process. Steps of fusion in medical image processing are presented in section VI. Section VII present some numerical experiments. We finally provide concluding remarks in Section VIII.

\section{PREVIOUS RELATED WORKS}

Many works have been done in the field of fuzzy information fusion in the literature. A brief review of some of them is presented in this section. Waltz [10] presented three basic levels of image data fusion : pixel level, feature level and decision level, which correspond to three processing architectures. I. Bloch [1] have outlined some features of Dempster-Shafer evidence theory, which can very useful for medical image fusion for classification, segmentation or recognition purposes. Examples were provided to show its ability to take into account a large variety of situations. Registration-based methods are considered as pixel-level fusion, such as MRI-PET (position emission tomography) data 
fusion[11]. Some techniques of knowledge-based segmentation can be considered as the feature-level fusion such as the methods proposed in [15].

Some belief functions, uncertainty theory, Dempster-Shafer theory are often used for decision-level fusion such as in [13]. In [16], I. Bloch proposed an unified framework of information fusion in the medical field based on the fuzzy sets, allow to represent and to process the numerical data as well as symbolic systems, the fuzzy sets theory is applied to three levels: at the low level to treat the basic numerical information contained in the images, as well as possible ambiguity between the classes; on the level object, to represent objects or structures in the images such as a fuzzy objects. at the higher level, to take into account a structural information and some characteristics as the distance, adjacency, and the relative position between objects.

V. Barra and J. Y. Boire [8] have described a general framework of the fusion of anatomical and functional medical images. The aim of their work is to fuse anatomical and functional information coming from medical imaging, the fusion process is performed in possibilistic logic frame, which allows for the management of uncertainty and imprecision inherent to the images. They particularly focus on the aggregation step with the introduction of a new class of operators based on information theory and the whole process is finally illustrated in two clinical cases: the study of Alzheimer's disease by MR/SPECT fusion and the study of epilepsy with MR/PET/SPECT. The obtained results was very encouraging.

V. Barra and J. Y. Boire [14] proposed a new scheme of information fusion to segment intern cerebral structures. The information is provided by MR images and expert knowledge, and consists of constitution, morphological and topological characteristics of tissues. The fusion of multimodality images is used in [12]. In [7], the authors have presented a framework of fuzzy information fusion to automatically segment tumor areas of human brain from multispectral magnetic resonance imaging (MRI) such as T1-weighted, T2-weighted and proton density (PD) images; in this approach three fuzzy models are introduced to represent tumor features for different MR image sequences. They allow to create corresponding fuzzy feature space of tumor. All the t-norm or fuzzy intersection operators can be used as fusion operators for this fuzzy features. the geometric mean is chosen using experiments allowing us to take correctly into account the three fuzzy spaces in a simple way. The fuzzy region growing is used to improve the fused result.

Maria del C. and al [9] proposed a new multispectral MRI data fusion technique for white matter lesion segmentation, in that a method is described and comparison with thresholding in FLAIR images is illustrated. Recently, The authors in [38] have presented a new framework of fuzzy information fusion using T2-weighted and proton density (PD) images to improve the brain tissue segmentation.

\section{THE FPCM AlgORITHM CLUSTERING}

Clustering is a process of finding groups in unlabeled dataset based on a similarity measure between the data patterns (elements) [16]. A cluster contains similar patterns placed together. One of the most widely used clustering methods is the FPCM algorithm. The FPCM algorithm solves the noise sensitivity defect of Fuzzy C-Means algorithm and overcomes the problem of coincident clusters of Possibilistic C-means algorithm. Given a set of $N$ data patterns $X=\left\{x_{1}, x_{2}, x_{3}, \ldots, x_{n}\right\}$ the Fuzzy Possibilistic C-Means (FPCM) clustering algorithm minimizes the objective function [31][32]:

$$
J(B, U, T, X)=\sum_{i=1}^{C} \sum_{j=1}^{N}\left(u_{i j}^{m}+t_{i j}^{\lambda}\right) d^{2}\left(x_{j}, b_{i}\right)
$$

Where $x_{j}$ is the $j$-th P-dimensional data vector, $b_{i}$ is the center of cluster $i, m>1$ is the weighting exponent, $\lambda \in[3,5]$ is the typicality exponent, $d^{2}\left(x_{j}, b_{i}\right)$ is the Euclidean distance between data $x_{j}$ and cluster center $b_{i},[U]_{C x N}$ is the fuzzy matrix and $[T]_{C X N}$ is the typicality matrix.

The minimization of objective function $J(B, U, T, X)$ can be brought by an iterative process in which updating of membership degrees $u_{i j}$, typicality degrees $t_{i j}$ and the cluster centers are done for each iteration by :

$$
\begin{gathered}
u_{i j}=\left[\sum_{k=1}^{c}\left(\frac{d^{2}\left(x_{j}, b_{i}\right)}{d^{2}\left(x_{j}, b_{k}\right)}\right)^{2 /(m-1)}\right]^{-1} . \\
t_{i j}=\left[\sum_{k=1}^{c}\left(\frac{d^{2}\left(x_{j}, b_{i}\right)}{d^{2}\left(x_{j}, b_{k}\right)}\right)^{2 /(\lambda-1)}\right]^{-1} . \\
b_{i}=\frac{\sum_{k=1}^{N}\left(u_{i k}^{m}+t_{i k}^{\lambda}\right) x_{k}}{\sum_{k=1}^{N}\left(u_{i k}^{m}+t_{i k}^{\lambda}\right)} .
\end{gathered}
$$

Where :

$$
\begin{gathered}
\forall i \in\{1 . . C\}, \quad \forall j \in\{1 . . N\} \quad\left\{\begin{array}{c}
u_{i j} \in[0,1] \\
0<\sum_{i=1}^{N} u_{i j}<N
\end{array} .\right. \\
\forall j \in\{1 . . N\} \quad \sum_{i=1}^{C} u_{i j}=1 . \\
\forall i \in\{1 . . C\} \quad \sum_{j=1}^{N} t_{i j}=1 .
\end{gathered}
$$

The algorithm of the FPCM consists then of the reiterated application of (2), (3) and (4) until stability of the solutions.

\section{THE POSSIBILITY THEORY}

Possibilistic logic was introduced by Zadeh (1978) following its former works in fuzzy logic (Zadeh, 1965) in order to simultaneously represent imprecise and uncertain knowledge. In fuzzy set theory, a fuzzy measure is a representation of the uncertainty, giving for each subset $\mathrm{Y}$ of the universe of discourse $X$ a coefficient in $[0,1]$ assessing the degree of certitude for the realization of the event $Y$. In possibilistic logic, this fuzzy measure is modeled as a measure of possibility $\Pi$ satisfying: 


$$
\begin{aligned}
& \Pi(X)=1 \quad \text { et } \quad \Pi(\phi)=0 \\
& \left(\forall\left(Y_{i}\right)\right) \Pi\left(\cup_{i} Y_{i}\right)=\operatorname{Sup}_{i} \quad \Pi\left(Y_{i}\right)
\end{aligned}
$$

An event $\mathrm{Y}$ is completely possible if $\Pi(Y)=1$ and is impossible if $\Pi(Y)=0$. Zadeh showed that $\Pi$ could completely be defined from the assessment of the certitude on each singleton of $X$. Such a definition relies on the definition of a distribution of possibility $\pi$ satisfying :

$$
\begin{aligned}
& \pi: X \rightarrow[0,1] \\
& x \rightarrow \pi(x) / \operatorname{Sup}_{x}\{\pi(x)=1\}
\end{aligned}
$$

Fuzzy sets $\mathrm{F}$ can then be represented by distributions of possibility, from the definition of their characteristic function $\mu_{F}$ :

$$
(\forall x \in X) \mu_{F}(x)=\pi(x)
$$

Distributions of possibility can mathematically be related to probabilities, and they moreover offer the capability to declare the ignorance about an event. Considering such an event A (e.g., voxel $\mathrm{v}$ belongs to tissue $\mathrm{T}$, (where $\mathrm{v}$ is at the interface between two tissues), the probabilities would assign $P(A)=P(\bar{A})=0.5$, whereas the possibility theory allows fully possible $\Pi(A)=\Pi(\bar{A})=1$. We chose to model all the information using distributions of possibility, and equivalently we represented this information using fuzzy sets [22].

The literature classically distinguishes three modes for combination of uncertainty and imprecise information in a possibility theory framework [27] :

The conjunction: gather the operators of t-norms (fuzzy intersection), this mode of combination must be used if measurements are coherent, i.e. without conflict.

The compromise: gather the median operator and some average operators, it must be used when measurements are in partial conflict.

The Disjunction: gather the operators of t-conorms (fuzzy union), it must be used when measurements are in disaccord, i.e. in severe conflict.

In introduction, we underlined the inopportunity to combining information in a fixed mode: if observations are in accord, it is legitimate to combine them in a conjunctive mode or compromise in order to extract a more relevant information. But if a serious conflict appears, it is better to combining in a disjunctive mode. For example, if two measurements of the same parameter prove completely different, it is not judicious to make an average of it, better is worth to say than one or the other is true [28].

\section{The Fusion Process AND TyPe OF ARCHITECTURES}

A general information fusion problem can be stated in the following terms: given $l$ sources $S_{l}, S_{2}, \ldots S_{l}$ representing heterogeneous data on the observed phenomenon, take a decision $d_{i}$ on an element $x$, where $x$ is higher level object extracted from information, and $D_{i}$ belongs to a decision space $D=\left\{d_{1}, d_{2}, d_{3}, \ldots, d_{n}\right\}$ (or set of hypotheses). In numerical fusion methods, the information relating $x$ to each possible decision $d_{i}$ according to each source $S_{j}$ is represented as a number $M_{i j}$ having different properties and different meanings depending on the mathematical fusion framework. In the centralized scheme, the measures related to each possible decision $i$ and provided by all sources are combined in a global evaluation of this decision, taking the form, for each $i: M_{i}=F\left(M_{i}, M_{i 2}, M_{i 3}\right.$, ..., $M_{\text {in }}$ ), where $F$ is a fusion operator. Then a decision is taken from the set of $M_{i}, 1 \leq i \leq n$. in this scheme, no intermediate decision is taken and the final decision is issued at the end of the processing chain. In decentralized scheme decisions at intermediate steps are taken with partial information only, which usually require a difficult control or arbitration step to diminish contradictions and conflicts [6][8].

The three-steps fusion can be therefore described as :

- Modeling of information in a common theoretical frame to manage vague, ambiguous knowledge and information imperfection. In addition, in this step the $M_{i j}$ values are estimated according to the chosen mathematical framework.

- Combination : the information is then aggregated with a fusion operator $F$. This operator must affirm redundancy and manage the complementarities and conflicts.

- Decision : it is the ultimate step of the fusion, which makes it possible to pass from information provided by the sources to the choice of a decision $d_{i}$.

\section{Data Fusion In IMage Processing Using POSSIBILITY THEORY}

\section{A. Modeling Step}

In the framework of possibility theory and fuzzy sets [17][18][19], the $M_{i j}$ 's represent membership degrees to a fuzzy set or possibility distribution $\pi$, taking the form for each decision $d_{i}$ and source $S_{i}: M_{i j}=\pi_{j}\left(d_{i}\right)$. Particularly, in our study this step consists in the creation of WM, GM, CSF and background (BG) fuzzy maps for both $\mathrm{T} 2$ and PD images using the FPCM algorithm then $u_{i j}=\pi_{j}\left(d_{i}\right)$

\section{B. Fusion step}

For the aggregation step in the fusion process, the advantages of possibility theory rely in the variety of combination operators, which must affirm redundancy and manage the complementarities. And may deal with heterogeneous information [20][21][22]. It is particular interest to note that, unlike other data fusion theories like Bayesian or

Dempster-Shafer combination, possibility theory provides a great flexibility in the choice of the operator, that can be adapted to any situation at hand [6]. If $\pi_{T}^{T 2}(v), \pi_{T}^{P D}(v)$ are the memberships of a voxel $v$ to tissue $T$ resulting from step 1 then a fusion operator $F$ generate a new membership value $\pi_{T}(v)=F\left(\pi_{T}^{T 2}(v),{ }_{T}^{P D}(v)\right)$ and can managing the existing 
ambiguity and redundancy. The possibility theory propose a wide range of operators for the combination of memberships. I. Bloch [24] classified these operators in three classes defined as:

- Context independent and constant behavior operators (CICB);

- Context independent and variable behavior operators (CIVB);

- Context dependent operators (CD).

For our MR images fusion, we chose a context-based conjunctive operator because in the medical context, both images were supposed to be almost everywhere concordant, except near boundaries between tissues and in pathologic areas [20]. In addition, the context-based behavior allowed to take into account these ambiguous but diagnosis-relevant areas. Then three operators of this class are chosen [23][24]:

$$
\begin{aligned}
& \text { FOP1 }: \pi_{T}(v)=\min \left(\pi_{T}^{T 2}(v), \pi_{T}^{P D}(v)\right)+1-h \\
& \text { FOP2 }: \pi_{T}(v)=\max \left(\frac{\min \left(\pi_{T}^{T 2}(v), \pi_{T}^{P D}(v)\right)}{h}, 1-h\right) \\
& \text { FOP3 }: \pi_{T}(v)=\min \left(1, \frac{\min \left(\pi_{T}^{T 2}(v), \pi_{T}^{P D}(v)\right)}{h}+1-h\right)
\end{aligned}
$$

Where $\mathrm{h}$ is a measure of agreement between $\pi_{T}^{T 2}$ and $\pi_{T}^{P D}$ :

$$
h=1-\sum_{v \in \operatorname{Im} a g e}\left|\pi_{T}^{T 2}(v)-\pi_{T}^{P D}(v)\right| / \mid \operatorname{Im} \text { age } \mid
$$

\section{Decision step}

A segmented image was finally obtained using the four maps computed in step 2 by assigning to the tissue $T$ any voxel for which it had the greatest degree of membership (i.e maximum of possibility rule)[6][23].

The general algorithm using for fusion process can be summarized as follows :

\section{General algorithm \\ Modeling of the image \\ For $\mathrm{i}$ in $\{\mathrm{T} 2, \mathrm{PD}\}$ do \\ FPCM (i) \{ Computation of membership degrees for both images $T 2$ and $P D$ \} \\ End For \\ Fusion \\ Possibilistic fusion $\{$ Between each class of T2 image and the same one of $P D$ image using FOP1, FOP2 or FOP3 operator $\}$}

\section{Decision}

Segmented image $\{$ maximum of possibility rule $\}$

It should be noted that the stability of this algorithm depend to the stability of the algorithm used in the modeling step[31][32].

\section{EXPERIMENTAL RESULTS}

Since the ground truth of segmentation for real MR images is not usually available, it is impossible to evaluate the segmentation performance quantitatively, but only visually. However, Brainweb[35] provides a simulated brain database (SBD) including a set of realistic MRI data volumes produced by an MRI simulator. These data enable us to evaluate the performance of various image analysis methods in a setting where the truth is known [34][35][36].

to have tests under realistic conditions, one volume was generated with a thickness of $1 \mathrm{~mm}$ and a level of noise of $3 \%$. We fixed at $20 \%$ the parameter of heterogeneity.

The results of each step of fusion on a noisy 94th brain only slice are shown in figure 1, 2 and 3. This noisy slice was segmented into four clusters: background, CSF, white matter, and gray matter using FPCM algorithm, however the background was neglected from the viewing results.

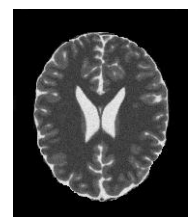

$94^{\text {th }}$ simulated T2 slice

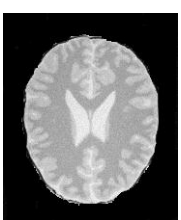

$94^{\text {th }}$ simulated PD slice (a)

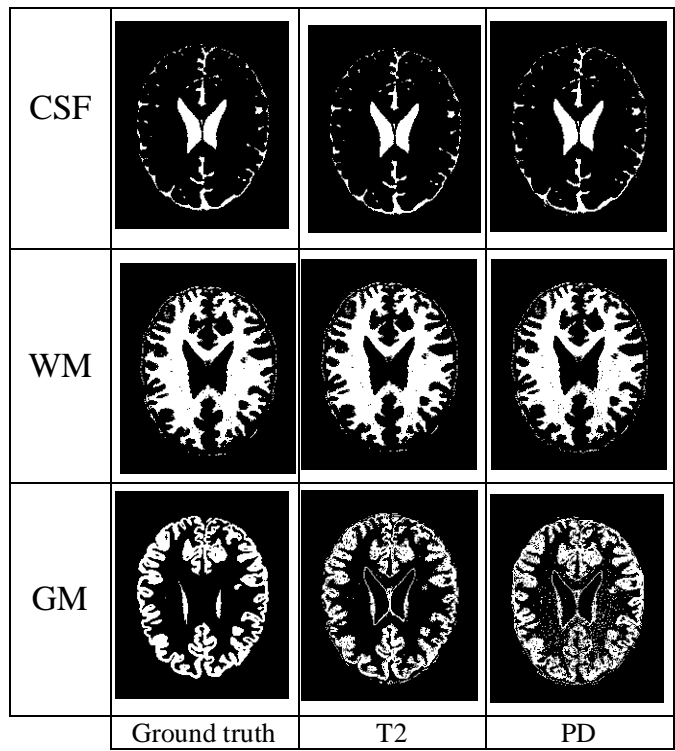

(b)

Figure 1. (a) Simulated T2, PD images illustrate the fusion. (b) Fuzzy maps of CSF, WM and GM obtained by FPCM algorithm accompagnied by the ground truth for T2 and PD image. 
The fused maps produced in fusion step using FOP1, FOP2 and FOP3 operators respectively are presented in figure 2 below :

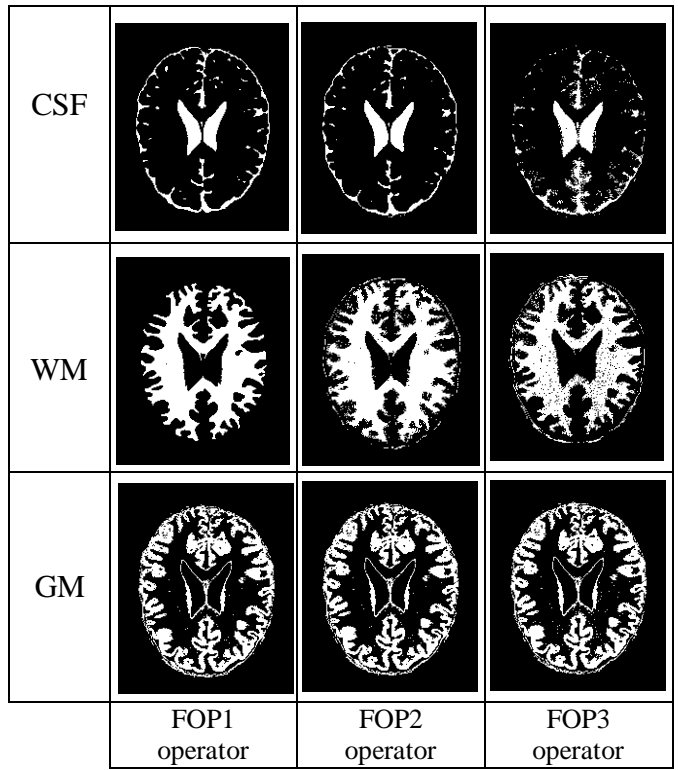

Figure 2. Fused maps obtained with different operators.

And the results of final segmentation with the different operators FOP1, FOP2 and FOP3 are shown in figure 3 below.

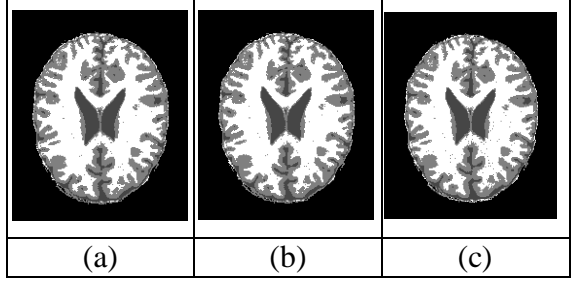

Figure 3. Final segmented images obtained by fusion. (a) FOP1 operator (b) FOP2 operator, (c) FOP3 operator.

The fusion with FOP1 operator improve significantly the CSF map of T2 and PD image.

The WM fused map is strongly improved compared to that obtained by the $\mathrm{T} 2$ only and the PD only.

Information in GM fused map with FOP1 operator is reinforced in area of agreement (mainly in the cortex). And the fusion showed a significant improvement and reduces the effect of noise in images.

To compare the performance of these various final segmentation produced by different operators, we compute different coefficients reflecting how well two segmented volumes match. We use a different performance measures[38]:

$$
\begin{gathered}
\operatorname{Overlap}(R E)=\frac{T P}{T P+F N+F P} . \\
\operatorname{Sensitivity}(S E)=\frac{T P}{T P+F N} .
\end{gathered}
$$

$$
\begin{gathered}
\text { Specificit } y(S P)=\frac{T N}{T N+F P} . \\
\operatorname{Similarity}(S I)=\frac{2 . T P}{2 . T P+F N+F P} .
\end{gathered}
$$

Where $T P$ and $F P$ stand for true positive and false positive, which were defined as the number of voxels correctly and incorrectly classified as brain tissue by the automated algorithm. $T N$ and $F N$ stand for true negative and false negative, which were defined as the number of voxels correctly and incorrectly classified as non-brain tissue by the automated algorithm. The results obtained by all operators are presented in figures 4, 5 and 6 below :

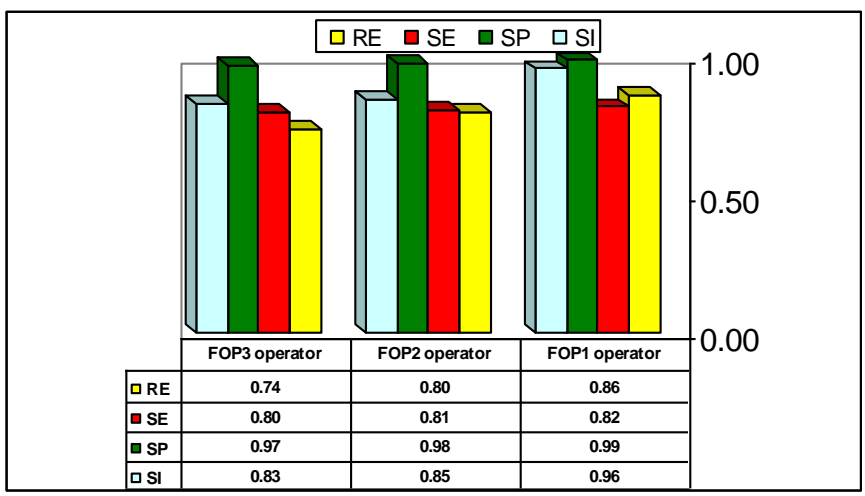

Figure 4. Performance measures of CSF mater.

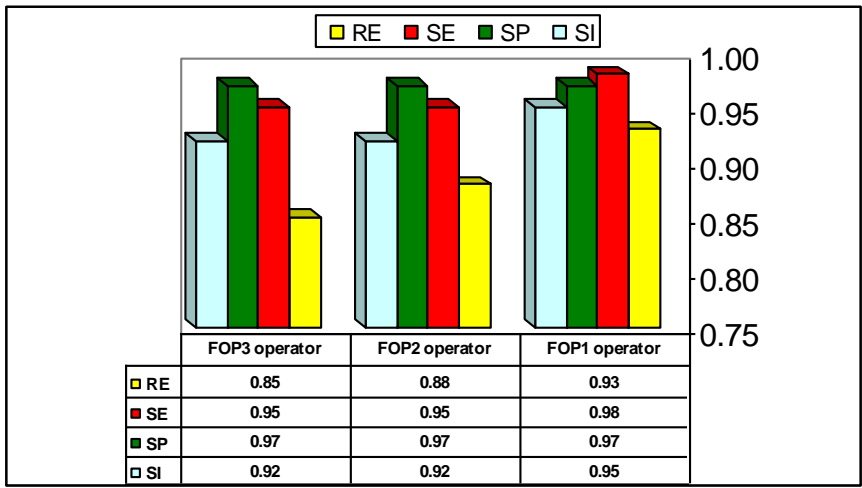

Figure 5. Performance measures of WM mater.

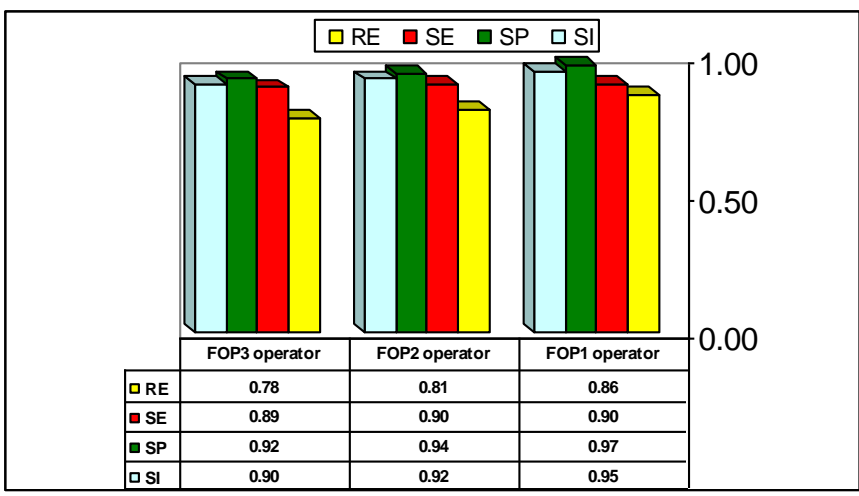

Figure 6. Performance measures of GM mater. 
The results showing in figures 4, 5 and 6 demonstrate easily the predominance of the FOP1 operator compared to both FOP2 and FOP3 operators, then the best segmentation is obtained by this one.

\section{A. Comparison with other methods :}

To validate the interest of fusion produced by operator FOP1 in terms of segmentation of the cerebral tissues, we compared the results obtained on fusion T2/PD with a fuzzy segmentation computed by the algorithm of classification FPCM on the T2 image alone and the PD image alone. An example of segmentation result for the slice 94 of Brainweb is presented in the figure 7 below:

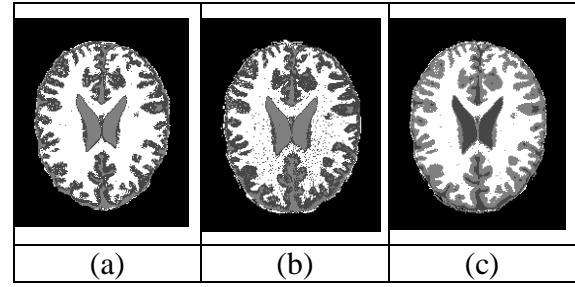

Figure 7. (a) T2 segmeneted with FPCM algorithm, (b) PD segmeneted with FPCM algorithm, (d) Image of fusion with FOP1 operator.

For each one of the segmentation, we calculated four measurements of validation: overlap, sensitivity, specificity and the similarity. For all tissues CSF, WM and GM. The results are reported in the figure 8,9 and 10 below :

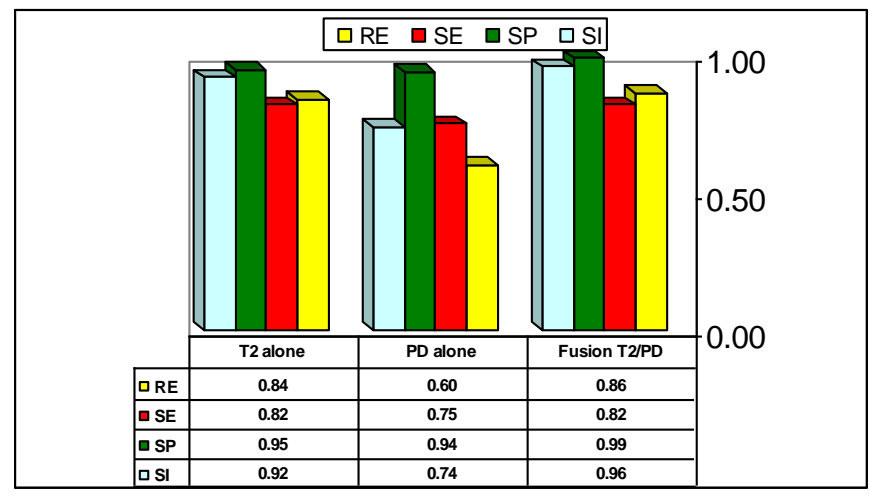

Figure 8. Performance measures of CSF mater.

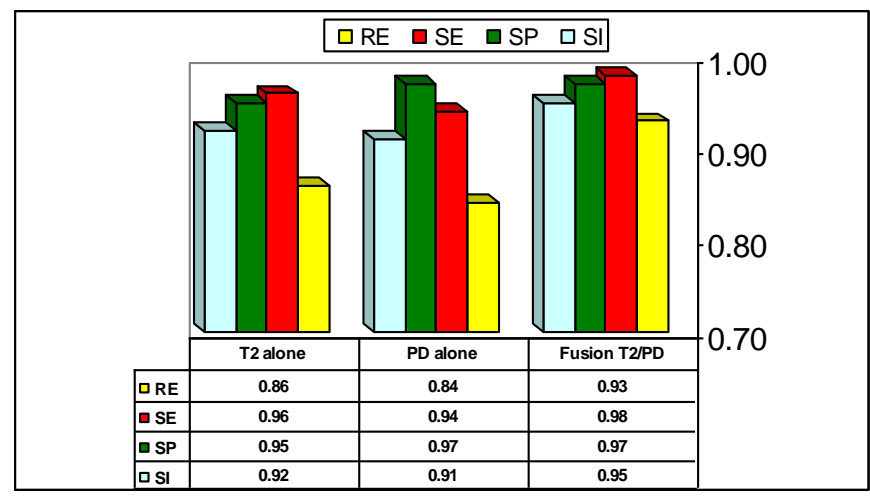

Figure 9. Performance measures of WM mater.

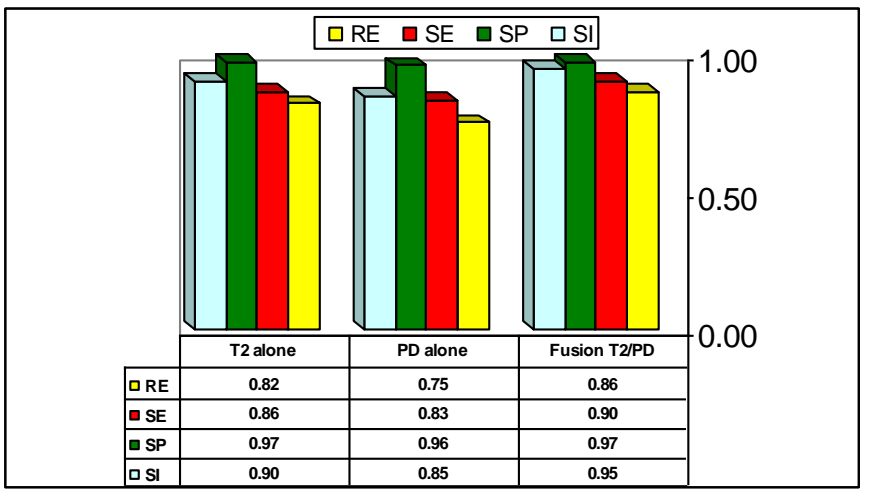

Figure 10. Performance measures of GM mater.

The graphics of figures 8,9 and 10 underline the advantages of the multispectral fusion images within the fuzzy possibilistic framework to improve the segmentation results clearly. Indeed all performance measurements obtained with fusion of T2 and PD images for all tissues CSF, WM and GM are greater than ones obtained when to taking into account of only one weighting in MR image segmentation.

\section{CONCLUSION}

This paper mainly focus on the study and evaluation of the segmentation of MR images with multispectral fusion approach using three different adaptive operators. We outlined in here some features of possibility theory, which can be very useful for medical images fusion. And which constitute advantages over classical theories. Our study demonstrate the superior capabilities of fusion approach compared to the taking into account of only one weighting in MR image segmentation.

As a perspective of this work other adaptive operators or more robust algorithms to representing a data are desired. In addition, we can integrate other numerical, symbolic information or images coming from other imaging devices include computer tomography(CT), the newer positron emission tomography (PET) or a major functional modality SPECT in order to improve the segmentation of the MR images or to detect anomalies in the pathological images.

\section{ACKNOWLEDGMENT}

The authors thank the reviewers for their valuable comments and suggestions that helped us to make the paper in its present form.

\section{REFERENCES}

[1] I. Bloch, "Some aspects of Dempster-Shafer evidence theory for classification of multi-modality medical images taking partial volume effect into account," Pattern Recognition Letters, vol. 17, pp. 905-919, 1996

[2] Y. Hata, S. Kobashi, and S. Hirano, "Automated segmentation of human brain MR images aided by fuzzy information granulation and fuzzy inference", IEEE Trans. SMC, Part C, vol. 30, pp. 381-395, 2000.

[3] D. Goldberg-Zimring, A. Achiron, and S. Miron, "Automated detection and characterization of multiple sclerosis lesions in brain MR images", Magnetic Resonance Imaging, vol. 16 pp. 311-318,1998.

[4] K. Van Leemput, F. Maes, D. Vandermeulen, and P. Suetens, "Automated model-based tissue classification of MR images of the brain”, IEEE Trans. Medical Imaging, vol. 18, pp. 897-908, 1999. 
[5] Y. Wang, T. Adali, J. Xuan, and Z. Szabo. "Magnetic resonance image analysis by information theoretic criteria and stochastic models", IEEE Trans, Information Technology in Biomedicine, vol. 5, pp. 150-158, 2001.

[6] I. Bloch, and H. Maitre, "Data fusion in 2D and 3D image processing: An overview," X Brazilian symposium on computer graphics and image processing, Brazil, pp. 127-134, 1997.

[7] W. Dou, S. Ruan, Y. Chen, D. Bloyet, J. M. Constans, "A framwork of fuzzy information fusion for the segmentation of brain tumor tissues on MR images," Image and vision Computing, vol. 25, pp. 164-171, 2007

[8] V. Barra and J. Y. Boire, "A General Framework for the Fusion of Anatomical and Functional Medical Images," NeuroImage, vol. 13, 410424, 2001.

[9] D. C. Maria, H. Valdés, J. F. Karen, M. C. Francesca, M. W. Joanna, "New multispectral MRI data fusion technique for white matter lesion segmentation: method and comparison with thresholding in FLAIR images," Eur Radiol, vol. 20, 1684-1691, 2010.

[10] E.D Waltz, the principals and practice of image and spatial data fusion in : Davis L, Hall, James llinas (Eds), Proceedings of the Eight, national Data Fusion Conference, (Dalls, TX. March 15-17, 1995) Handbook of Multisensor Data Fusion, CRC pess, West Bam Beach, FL,1995, pp. 257-278. (p4-1-4-18).

[11] F. Behloul, M. Janier, P. Croisille, C. Poirier, A. Boudraa, R. Unterreiner, J. C. Mason, D. Revel, "Automatic assessment of myocardial viability based on PET-MRI data fusion," Eng. Med. Biol. Mag., Proc. 20th Ann. Int. Conf. IEEE, vol. 1, pp. 492-495, 1998.

[12] M. Aguilar, R. Joshua, "New fusion of multi-modality volumetric medical imagery," ISIF, pp. 1206-1212, 2002.

[13] E. Lefevre, P. Vannoorenberghe, O. Colot, "About the use of DempsterShafer theory for color image segmentation," First international conference on color in graphics and image processing, Saint-Etienne, France, 2000.

[14] V.Barra, and J-Y Boire, "Automatic segmentation of subcortical brain structures in MR images using information fusion," IEEE Trans. on Med. Imaging., vol. 20, n7, pp. 549-558, 2001.

[15] M. C. Clarck, L.O. Hall, D.B. Goldgof, R Velthuizen, F.R. Murtagh, M.S. Silbiger, "Automatic tumor segmentation using knowledge-based techniques,” IEEE Trans. Med. Imaging, vol. 17, pp. 187-201, 1998.

[16] I. Bloch, "Fusion of numerical and structural image information in medical imaging in the framework of fuzzy sets," Fuzzy Systems in Medicine, ed. par P. Szczepaniak et al., pp. 429-447, Springer Verlag, 2000.

[17] L. A. Zadeh, "Fuzzy sets," Information and Control, vol. 8, pp. 338-353, 1965.

[18] L. A. Zadeh, "fuzzy sets as a basic for a theory of possibility," Int. Jour. of Fuzzy Sets and Systems, vol. 1, pp. 3-28, 1978.

[19] D. Dubois and H. Prade. Fuzzy sets and systems: Theory and Application, New-York: Academic Press, 1980.

[20] D. Dubois and H. Prade, "A review of Fuzzy Set Aggregation Connectives," Information Sciences, vol. 36, pp. 85-121, 1985.

[21] R. R. Yager, "Connectives and quantifiers in fuzzy sets," Int. Jour. of Fuzzy sets and systems, vol. 40, pp. 39-75,1991.

[22] D. Dubois and H. Prade, "Combination of information in the framework of possibility theory," In M. AL Abidi et al., editor, Data Fusion in Robotics and Machine Intelligence: Academic Press, 1992.

[23] V. Barra, Fusion d'images 3D du cerveau : Etude de modèles et Applications. Thèse de doctorat, Université d'Auvergne, 2000.

[24] I. Bloch, "Information combination operators for data fusion: A Comparative Review with Classification," IEEE Transactions en systems, Man. and Cybernitics, vol. 1, pp. 52-67, 1996.

[25] W. Dou, Segmentation des images multispectrales basée sur la fusion d'information: application aux images IRM. Thèse de doctorat Université CAEN/Basse-Normandi , 2006.

[26] A.Bendjebbour, W.Pieczynski, "Segmentation d'images multisenseurs par fusion évidentielle dans un contexte markovien," Traitement du Signal, vol. 14, n15, pp. 453-463, 1997.
[27] H.J. Zimmermann. Fuzzy Sets Theory and its Applications. 2nd ed. Boston: Kluwer Academic Publishers, 1991.

[28] S. Deveughelle, B. Dubuisson, "Adaptabilité et combinaison possibiliste: application à la vision multi caméras," Traitement du Signal, vol. 11, n6, pp. 559-568, 1994.

[29] C.Barillot, J.C.Gee,L.Le Briquer,G.Le Goualher, "Fusion intra et inter individus en imagerie médicale appliquée à la modélisation anatomique du cerveau humain," Traitement du Signal, vol. 11, n6, 1994.

[30] J. Bezdek, "A convergence theorem for the fuzzy data clustering algorithms," IEEE Transactions on Pattern Analysis and Machine Intelligence TPAMI, vol. 2, pp. 1-8,1980.

[31] J. C. Bezdek, J. Keller, R. Krishnapuram and N. R. Pal, "Fuzzy Models and Algorithms for Pattern Recognition and Image Processing", Kluwer Academic Publishers, TA 1650.F89, 1999.

[32] N. R. Pal, K. Pal and J. C. Bezdek, "A mixed c-means clustering model", Proceedings of the Sixth IEEE International Conference onFuzzy Systems, Vol. 1, pp. 11-21, 1997.

[33] V.Barra, J. H. Boire, Segmentation floue des tissus cérébraux en IRM 3D: une approche possibiliste versus autres méthodes, Rencontres Francophones sur la logique floue et ses applications, Valenciennes, Editions Cépaduès, pp. 193-198, 1999.

[34] I.Bloch, H. Maitre, "Fusion de données en traitement d'images : modèles d'informations et décisions," Traitement du Signal, vol. 11, n6, pp. 435446, 1994.

[35] BrainWeb [Online]. Available: www.bic.mni.mcgill.ca/brainweb/

[36] C. A. Cocosco, V. Kollokian, R. K. -S. Kwan, and A. C. Evans, "BrainWeb: Online interface to a 3D MRI simulated brain database," NeuroImage, pt. 2/4, vol. 5, n4, p. S425, 1997.

[37] D. L. Collins, A. P. Zijdenbos, V. Kollokian, J. G. Sled, N. J. Kabani, C. J. Holmes, and A. C. Evans, "Design and construction of a realistic digital brain phantom," IEEE Trans. Med. Imaging, vol. 17, n3, pp. $463-$ 468, 1998.

[38] C.Lamiche, A.Moussaoui, "Improvement of brain tissue segmentation using information fusion approach", International Journal of Advanced Computer Science and Applications, vol 2, n6, pp. 84-90, 2011.

[39] N. Richard, M. Dojat, and c. garbay, "Automated segmentation of humain brain MR images using a multi-agent approach", Artificial intelligence in Medecine, 2004.

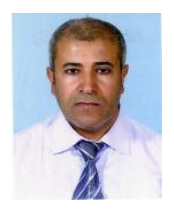

\section{AUTHORS PROFILE}

Lamiche Chaabane received his computer science engineer degree in 1997 from the Department of Computer Science from Ferhat Abbas University, Algeria. He also received a Magister degree in Computer Science in 2006 from University of M'sila. He has 4 years experience in teaching. His areas of interests include data mining and warehousing, artificial intelligence, image processing and operational research. His current research interests include the data mining techniques and medical image analysis.

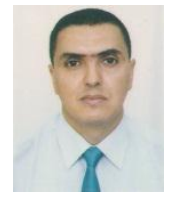

Moussaoui Abdelouahab received his BSc in Computer Science in 1990 from the Department of Computer Science from the University of Science and Technology of Houari Boumedienne (USTHB), Algeria. He also received a MSc in Space Engineering in 1991 from University of Science and Technology of Oran (USTO). He received also a MSc degree in Machine Learning from Reims University (France) since 1992 and Master's degree in Computer Science in 1995 from University of Sidi Bel-abbes, Algeria and $\mathrm{PhD}$ degree in Computer Science from Ferhat Abbas University, Algeria. He is IEEE Member and AJIT, IJMMIA \& IJSC Referee. His researches are in the areas of clustering algorithms and multivariate image classification applications. His current research interests include the fuzzy neuronal network and non parametric classification using unsupervised knowledge system applied to biomedical image segmentation. He also works from a long time on pattern recognition's algorithm, complex data mining and medical image analysis. 